\title{
El coste del proceso de cambio de útiles
}

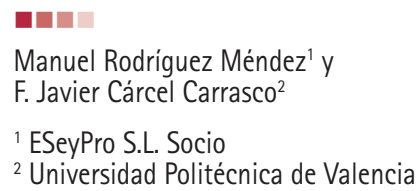

\section{INTRODUCCIÓN}

La producción es una red de operaciones y procesos, entrelazados en el espacio y en el tiempo, que permiten transformar unas materias primas en productos con una utilidad determinada. Todo este entramado de operaciones y procesos está sometido a criterios de costes, eficiencia, calidad, sostenibilidad y seguridad. Uno de esos procesos que configura esta red se conoce como cambio de útiles y se refiere al conjunto de operaciones necesarias para preparar los medios de producción y poder elaborar en ellos un producto diferente al que ya estaban elaborando.

El cambio de útiles, históricamente, se ha realizado previa una parada de los medios de producción, de ahí que siempre se le ha considerado una "operación de despilfarro" debido a que se realizaba con los medios de producción parados [1]. La producción basada en elevados tamaños de lotes diluía el problema que esta operación generaba, ya sea no considerando el coste de esta operación, o bien dándole un valor estándar a su tiempo y coste. Sin embargo, a medida de que se iba disminuyendo el tamaño del lote de producción, la relevancia que el cambio de útiles iba adquiriendo dentro del entramado productivo se iba haciendo cada vez mayor. Fue entonces cuando la gestión de la producción dedicó esfuerzos a minimizar el efecto del cambio de útiles con el objetivo de minimizar el tiempo de parada de los medios de producción [2]. El modelo SMED (Single Minute Exchange of Die) de Shigeo Shingo [3] siempre ha sido el modelo de referencia para minimizar el tiempo de parada de máquinas hasta lograr tiempos por debajo de los 10 minutos, desarrollando los conceptos de operación interna (la que se realizaba con los medios de producción parados) y operación externa (la que se puede realizar con los medios de producción en marcha). No obstante, Shigeo Shingo tratando de reducir aún más el tiempo de parada para el cambio de útiles desarrollo, posteriormente, el modelo OTED (One-Touch Exchange of Die) para acercarse al objetivo de no tener que parar los medios de producción basándose en mejoras técnicas y en la transformación de las operaciones que configuran el cambio de útiles en operaciones externas.

La irrupción de nuevas variables en la gestión de la producción más allá de la rentabilidad económica, tales como la calidad del producto, la seguridad laboral de las personas que participan en los procesos y, también, el medioambiente hicieron que el cambio de útiles también se viera involucrado en esta nueva forma de gestión, siendo la seguridad laboral la que más ha condicionado la realización del cambio de útiles al no permitir un acceso más libre y directo sobre las máquinas durante su etapa productiva, impidiendo, en algunos casos, la conversión de operaciones internas en operaciones externas, conforme la denominación acuñada gracias al modelo SMED de mejora de este proceso [4-6]. En consecuencia, el tiempo dejó de ser la variable por excelencia, asociada al estudio y mejora del cambio de útiles, y pasó a compartir su relevancia con el coste de este proceso $[7,8]$.

Los primeros estudios de costes de producción asociados al cambio de útiles sirvieron para relacionar este parámetro con el tamaño óptimo del lote de producción, mediante la Ecuación de Wilson $[9,10]$. En la última década del siglo XX ya toman relevancia los estudios del cambio de útiles que contemplan de forma conjunta su tiempo y coste de realización como los parámetros más relevantes a considerar durante esta etapa del proceso productivo global [11-14].

Este artículo aborda el estudio de los costes de un proceso de cambio de útiles desde la variabilidad que supone la realización de este proceso ya que es altamente dependiente de la forma, ta- maño y características físico-químicas de los productos que se procesan, de las personas que intervienen en el proceso de cambio, como de los equipos que se emplearán durante dicho proceso. Se presenta un programa de cálculo utilizado tanto para evaluar el coste de este proceso como valor de referencia en las etapas de mejora del cambio de útiles; desglosando su coste según las operaciones internas y externas descritas por Shigeo Shingo e introduciendo los costes de amortización, mano de obra y de oportunidad generados por el proceso.

\section{DESARROLLO DELL COSTE DEL CAMBIO DE ÚTILES}

El coste de un proceso de cambio de útiles es un parámetro que permite estimar el tamaño económico del lote de producción, pero, además, permite asociar su valor al coste de la flexibilidad del proceso productivo de una empresa $\mathrm{y}$, en resumen, va a permitir poder calcular el coste asociado a un producto $[15,16]$. El coste del proceso de cambio de útiles posee, además, otra característica que lo diferencia del coste de producción asociado a las máquinas que es su variabilidad.

Pues si el coste de producción de las máquinas suele ser constante, al poder serlo también sus velocidades de trabajo, el coste del proceso de cambio de útiles no tiene porqué serlo ya que depende de los tipos de cambio que se realicen en las máquinas [17].

En base a la tipología de operaciones propuestas por Shigeo Shingo, el coste de un proceso de cambio de útiles viene determinado por la suma de los costes de las operaciones externas (operaciones realizadas con los medios de producción en fase productiva) y de las operaciones internas (con los medios de producción parados). Por lo que el coste total del proceso se puede expresar mediante la fórmula

$$
\mathrm{C}_{\mathrm{T}}=\mathrm{C}_{\mathrm{INT}}+\mathrm{C}_{\mathrm{EXT}}
$$

Donde $\mathrm{C}_{\mathrm{T}}$ es el coste total, $\mathrm{C}_{\mathrm{INT}}$ es el coste de las operaciones internas y $\mathrm{C}_{\mathrm{EXT}}$ es el coste de las operaciones externas. 
En los epígrafes siguientes, de este mismo capítulo, se propone la forma de estimar cada uno de estos parámetros.

\subsection{EL COSTE DE LAS OPERACIONES EXTERNAS}

Este tipo de operaciones son aquellas que se realizan cuando el proceso de producción está en marcha, y su coste está asociado a los trabajadores que están realizando las actividades de preparación de las máquinas. En este apartado, se debe de incluir el coste de todas aquellas actividades que se realizan sobre las máquinas cuando éstas aún están en fase de producción. Algunas de estas actividades son:

- La organización del proceso.

- El desplazamiento al almacén de útiles para recoger los nuevos elementos a introducir en las máquinas.

- El desplazamiento desde las máquinas al almacén de útiles para llevar los elementos retirados de las máquinas.

- La limpieza de útiles.

- El transporte de herramientas.

- El coste de la mano de obra para la realización del intercambio de piezas en las máquinas.

También se debe de incluir en este apartado el coste de las mejoras realizadas para convertir las operaciones internas en externas.

\subsubsection{El coste de la organización} del cambio de útiles

Cualquier producto, que el departamento de producción tiene necesidad de elaborar, surge de una necesidad que el mercado tiene de él. Esta necesidad, en cualquier empresa, antes de llegar a la fase de producción, circula como información por diversos estamentos dentro de la empresa hasta llegar a iniciarse su elaboración. Esa necesidad que el mercado tiene de un producto entra en la empresa y, hasta que ese producto empieza a producirse en el departamento de producción, genera un conjunto de acciones, sobre todo de información, encaminadas a preparar los medios de producción para elaborar el producto demandado. Esta información que circula por la empresa genera un proceso de organización para la preparación de las máquinas.
El coste de este proceso organizativo se puede estimar mediante la ecuación:

$$
\mathrm{C}_{\mathrm{ORG}}=\sum_{\mathrm{i}=1}^{\mathrm{n}} \mathrm{C}_{\mathrm{i}} \times \mathrm{t}_{\mathrm{i}}
$$

Donde $\mathrm{C}_{\mathrm{ORG}}$ es el coste total de la organización del proceso de cambio de útiles, $\mathrm{C}_{\mathrm{i}}$ es el coste del operario i que interviene en cada una de las actividades implicadas en la organización del proceso de cambio de útiles, $t_{i}$ es el tiempo asignado al operario i que interviene en cada una de las actividades desarrolladas para organizar el proceso de cambio de útiles y $\mathrm{n}$ es el número total de operarios que intervienen en la organización del proceso de cambio de útiles.

2.1.2. El coste de la operación de preparación del cambio de útiles

La parte inicial del proceso de cambio de útiles lo configura las operaciones de preparación de los útiles que se van a colocar en las máquinas. Estos útiles, que se suponen ordenados en el lugar de almacenamiento, hay que localizarlos, recogerlos y transportarlos hacia el lugar donde están las máquinas donde se van a utilizar. Luego hay que colocarlos en las máquinas y, una vez terminada la producción, habrá que devolverlos a su lugar de almacenamiento, previa labor de inspección de su estado y limpieza. La ubicación ordenada de los útiles en su lugar de almacenamiento permitirá una rápida y fácil localización y, por supuesto, llevarlos rápidamente a su lugar de uso.

Básicamente, todas las actividades implicadas en este epígrafe se pueden agrupar en el siguiente conjunto de operaciones:

1. Operaciones de desplazamiento del operario, como por ejemplo:

1. Desde la máquina al almacén de útiles.

2. Transporte de las piezas desde el lugar de almacenamiento hasta la máquina donde se van a utilizar.

3. Transporte de las piezas retiradas de la máquina hasta su lugar de almacenamiento.

4. Volver a su puesto de trabajo.

2. Operaciones que el operario realiza sin realizar desplazamientos, como por ejemplo:
1. Recogiendo las piezas del almacén y colocándolas en su medio de transporte, cuando proceda.

2. Retirando las piezas de las máquinas y colocándolas en su medio de transporte para llevarlas a su lugar de almacenamiento.

3. Colocación de las piezas en las máquinas para la nueva etapa productiva.

4. Colocación de las piezas en su lugar de almacenamiento.

3. La inspección y limpieza de las piezas retiradas de las máquinas. Esta operación, que en principio podía incluirse en alguna clasificación anterior, es importante ya que puede implicar la eliminación o no de una pieza defectuosa y, posteriormente, su sustitución.

Durante esta etapa de preparación, hay actividades que implican movimiento por parte de quien las realiza y otras en las que el que las realiza no se desplaza. El coste de todas estas actividades se puede estimar mediante la ecuación:

$$
\mathrm{C}_{\mathrm{P}}=\sum_{\mathrm{i}=1}^{\mathrm{n}}\left(\mathrm{tm}_{\mathrm{i}}+\mathrm{tp}_{\mathrm{i}}+\mathrm{tr}_{\mathrm{i}}\right) \times \mathrm{C}_{\mathrm{i}}+\mathrm{C}_{\mathrm{m}}
$$

Donde $\mathrm{C}_{\mathrm{p}}$ es el coste total de las operaciones de preparación de los útiles, $\mathrm{tm}_{\mathrm{i}}$ es el tiempo en que el operario i está en movimiento, tp $_{i}$ es el tiempo en que el operario i no está efectuando desplazamiento, $\mathrm{tr}_{\mathrm{i}}$ es el tiempo de revisión de piezas, realizado por el operario $i, \mathrm{C}_{\mathrm{i}}$ es el coste horario de los operarios i y $\mathrm{C}_{\mathrm{m}}$ es el coste de mejora para transformar las operaciones internas en operaciones externas.

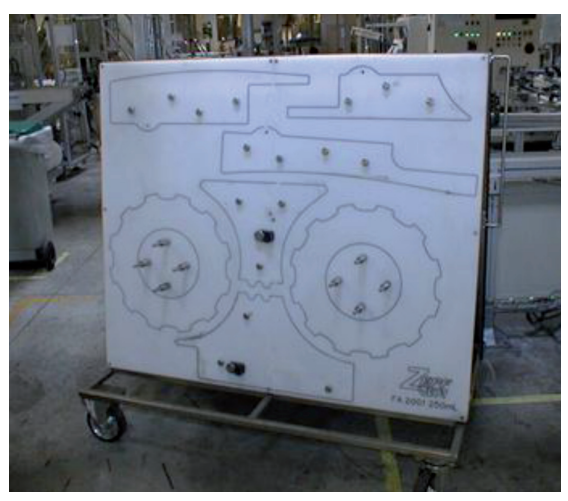

Fig.1: Elemento de transporte de útiles

Los tiempos en donde el operario está en movimiento, tm, se les puede asignar un valor constante, ya que en 
un proceso de cambio de útiles, adecuadamente organizado, la separación entre los almacenes de materiales y las máquinas no suele sufrir grandes variaciones. Sin embargo, los otros tiempos asignados a tp y tr, no tienen por qué ser constantes, ya que, dependen del tipo de cambio de útiles que se vaya a realizar, de la semejanza de formatos entre el producto que está en la máquina y el producto al que se va a cambiar ya que esto condicionará el tipo de piezas que se vayan a cambiar y, por lo tanto, también el número de piezas a recoger $\mathrm{y}$, también, el tiempo necesario para ello.

\subsection{EL COSTE DE LAS}

\section{OPERACIONES INTERNAS}

La etapa más importante del cambio de útiles es el intercambio de piezas en las máquinas que se realiza, generalmente, con la línea de producción parada. Este intercambio, condicionado por el modo de ejecución de las etapas anteriores, viene determinado por el coste de la mano de obra implicada en esta operación, y por el coste de oportunidad que ella genera $[18,19]$. En este grupo de operaciones tenemos:

- La retirada, en las máquinas, de las piezas que no se van a utilizar.

- La colocación, en las máquinas, de las piezas necesarias para procesar el nuevo producto.

- Las operaciones de ajuste de piezas ya colocadas en las máquinas.

- Las operaciones de adaptación de la máquina a las dimensiones y otras características físicas y físico-químicas del nuevo producto a elaborar.

Este coste viene dado por el coste de la mano de obra de las personas que intervienen en la realización de estas operaciones y también el coste de oportunidad generado.

2.2.1. El coste de la mano de obra

El cálculo del coste de la mano de obra que realiza el cambio de útiles, se calcula mediante la Ecuación

$$
\mathrm{C}_{\mathrm{p}}=\sum_{\mathrm{i}=1}^{\mathrm{n}} \mathrm{t}_{\mathrm{i}, \mathrm{j}} \times \mathrm{t}_{\mathrm{i}, \mathrm{j}}
$$

En donde $\mathrm{C}_{\mathrm{p}}$ es el coste total de todos los operarios que interviene en el proceso de cambio de útiles mientras las máquinas están paradas, $\mathrm{t}_{\mathrm{i}, \mathrm{j}}$ es el tiempo que cada operario, $\mathrm{i}$, dedica a realizar la operación $\mathrm{j}$ del proceso de cambio y $\mathrm{C}_{\mathrm{i} j}$ es el coste del operario i que realiza la operación j.

\subsubsection{Desarrollo del coste de} oportunidad

El coste de oportunidad que se genera durante el cambio viene dado por el beneficio que la empresa está perdiendo al no poder poner en el mercado el producto que se podía elaborar durante el tiempo de parada de los medios de producción por estar realizando en ellos el proceso de cambio de útiles. También se debe de considerar el coste de la mano de obra de producción que permanece sin ocupar, a la espera de estar la máquina en condiciones de trabajo $\mathrm{y}$, además, hay que considerar los costes de amortización de la máquina durante el tiempo de parada. En algunos casos, se ha de considerar el coste del capital invertido en las materias primas que están a la espera de entrar en el proceso de fabricación.

\section{RESULTADO: UN PROGRAMA DE CÁLCULO APLICADO A UNA INDUSTRIA}

Esta estructura de costes aquí presentada, se ha aplicado a una empresa del sector cosmético tanto para estimar el coste del proceso y asociarlo al producto, como en las etapas de mejora del cambio de útiles, mostrándose como una herramienta eficaz para dosificar los esfuerzos de mejora de los medios de producción a las exigencias del mercado. Para ello se ha desarrollado un programa de cálculo que permite estimar el coste de un proceso de cambio de útiles. Este programa, desarrollado en FORTRAN, consta de un programa ejecutable donde, además de recibir todos los parámetros necesarios para realizar los cálculos, estima los costes de las operaciones externas y de la mano de obra que interviene en las operaciones internas. Este programa principal se apoya en dos subrutinas en donde se calculan los costes de oportunidad y los de amortización del proceso; ambos costes, luego, son enviados al programa principal.

El programa ejecutable se apoya en diversos archivos donde lee los datos referidos a los costes de cada uno de los operarios de la empresa, donde el ejecutable va a buscar el parámetro asociado a las personas que intervienen en la organización y a cada etapa y operación del proceso de cambio de útiles. También, se podrá leer los valores de amortización de los equipos y costes de las herramientas empleadas en cada uno de los procesos. En la Figura 3 se muestra el diagrama de flujo del programa principal.

La salida de datos, Figura 2, se hace en un fichero en donde se muestra, por separado, los costes de mano de obra directa e indirecta, el coste de la organización puesta en marcha para el proceso de cambio de útiles y, también, el coste de oportunidad. Con todos estos datos, el programa principal puede estimar el coste total del proceso de cada cambio de útiles.

La Figura 4 muestra el diagrama de flujo de la subrutina que realiza la estimación del coste de oportunidad generado por estar realizando el proceso de cambio de útiles, para lo cual es necesario estimar los costes de amortización, de los equipos y herramientas, que se realiza con la subrutina cuyo diagrama de flujo se muestra en la Figura 5.
COSTE TOTAL DEL CAMBIO DE FORMATO

OPERACIONES EXTERNAS

Coste de organización

Coste de preparación

45,24

OPERACIONES INTERNAS:

Coste de mano de obra

Coste de oportunidad

COSTE TOTAL DEL CAMBIO DE FORMATO: $\quad 167,01$

Fig. 2: Formato de la salida de datos 
El programa presentado en este artículo sólo estima el coste del proceso de cambio de útiles, y queda para trabajos futuros el poder desarrollar una aplicación informática que permita establecer una relación directa entre este coste y el tamaño óptimo del lote de producción y el coste de elaboración del producto.

\section{CONCLUSIONES}

Este trabajo presenta una metodología de cálculo del coste del cambio de útiles utilizando como base la terminología desarrollada en el modelo SMED de mejora del cambio de útiles. Sin embargo introduce variables tales como el coste de la organización y preparación del cambio de útiles, el coste de oportunidad generado en el proceso y el coste de amortización de los elementos que intervienen en él y que no son tenidas en cuenta, o mencionadas, en la bibliografía existente sobre el cambio de útiles [20-22].

El método descrito en el presente artículo, como se ha dicho, se aplicó a una empresa del sector cosmético tanto para estimar el coste del proceso como en los procesos de mejora del cambio de útiles, mostrándose como una herramienta eficaz para dichos propósitos y ha permitido dosificar los esfuerzos de mejora del cambio de útiles. En la Tabla 1 se presenta la estimación del coste de un proceso de cambio de útiles durante las etapas de mejora del proceso. Las etapas 1, 2 y 3 corresponden, básicamente a las mejoras introducidas en la mejora de la organización del proceso de cambio y en el almacenamiento y transporte de los útiles, Figura 1, aunque también hay alguna mejora técnica, y las etapas 4, 5 y 6 corresponden, básicamente, a mejoras técnicas realizadas acompaña-

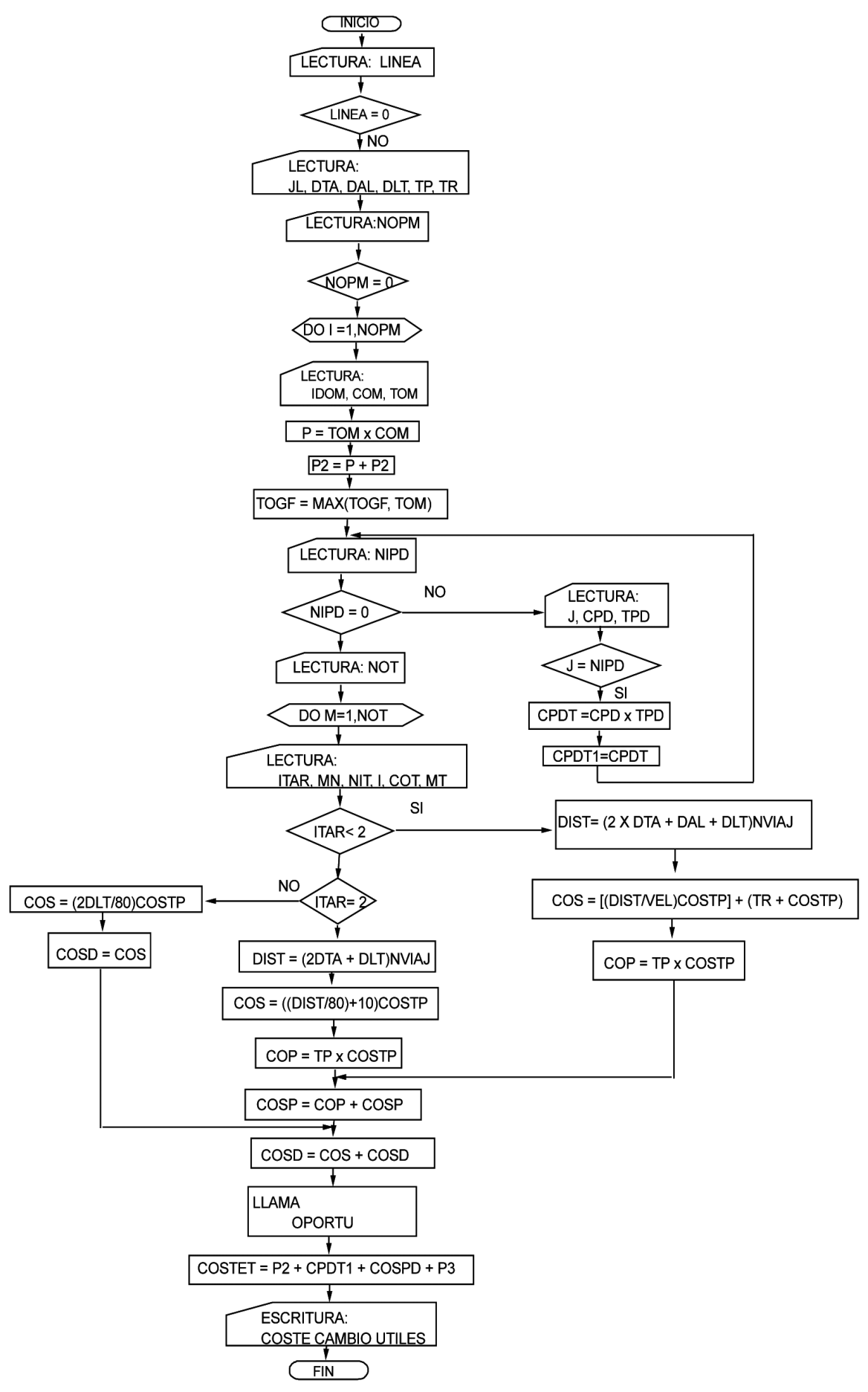

Fig. 3: Diagrama de flujo del programa principal

\begin{tabular}{|c|c|c|c|c|c|c|c|}
\hline & & \multicolumn{6}{|c|}{ COSTE $(€)$} \\
\hline \multicolumn{2}{|c|}{ Fases de la etapa de mejora } & 1 & 2 & 3 & 4 & 5 & 6 \\
\hline \multirow{2}{*}{ 0. EXTERNAS } & Coste de organización & 34,24 & 13,22 & 10,06 & 9,41 & 8,90 & 8,50 \\
\hline & Coste de preparación & - & 34,25 & 16,13 & 13,50 & 12,70 & 12,50 \\
\hline \multirow{2}{*}{ 0. INTERNAS } & Coste mano de obra & 140,48 & 84,31 & 63,31 & 25,70 & 20,30 & 16,20 \\
\hline & Coste de oportunidad & 145,34 & 75,31 & 49,10 & 41,44 & 39,80 & 28,30 \\
\hline \multicolumn{2}{|c|}{ COSTE TOTAL DEL PROCESO DE CAMBIO DE ÚTILES (€) } & 320,06 & 207,09 & 138,60 & 90,05 & 81,70 & 65,50 \\
\hline \multicolumn{2}{|c|}{ Tiempo cambio de útiles (h.) } & 6,54 & 4,33 & 2,36 & 1,16 & 0,93 & 0,47 \\
\hline
\end{tabular}

Tabla 1: Presentación de resultados utilizados durante una fase de mejora en industria cosmética 
das de ya algunas mejoras de carácter organizativo.

Este artículo presenta las pautas para estimar, de forma sencilla, el coste de un proceso de cambio de útiles que se puede emplear tanto en la etapa de producción diaria, para estimar el coste del proceso productivo total, sin necesidad de recurrir a la estandarización del coste de cambio de útiles, como en las fases de mejora del proceso de cambio de útiles, para dosificar los esfuerzos de la empresa en la adaptación de sus tamaños de lote de producción a las necesidades del mercado. Además, con este programa y con esta forma de desglosar el coste, los gestores de producción podrán dirigir los esfuerzos de mejora a aquellas actividades que configuran el cambio de útiles, sin necesidad de alterar el orden de los lotes a producir para conseguir una minimización global de los costes [23] que obligue a repartir el coste del cambio de útiles entre los lotes a producir por incapacidad de asignar el coste correcto a cada lote.

Queda para proyectos futuros el asociar el programa de cálculo del coste a otras aplicaciones que permitan obtener los tiempos de parada de máquinas y la captura de datos de las personas que participan en esta actividad, para poder estimar, in situ, el coste de forma inmediata.

\section{AGRADECIMIENTOS}

Deseamos agradecer a todos los anónimos revisores de este artículo todos sus comentarios ya que nos han permitido mejorar su contenido.

\section{PARA SABER MÁS}

[1] Moon I, Giri B C, Choi K. "Economic lot scheduling problem with imperfect production processes and setup times." Journal of the Operational Research Society. 2002. Vol. 53-6. p. 620-629. D0I:10.1057| palgrave.jors. 2601350

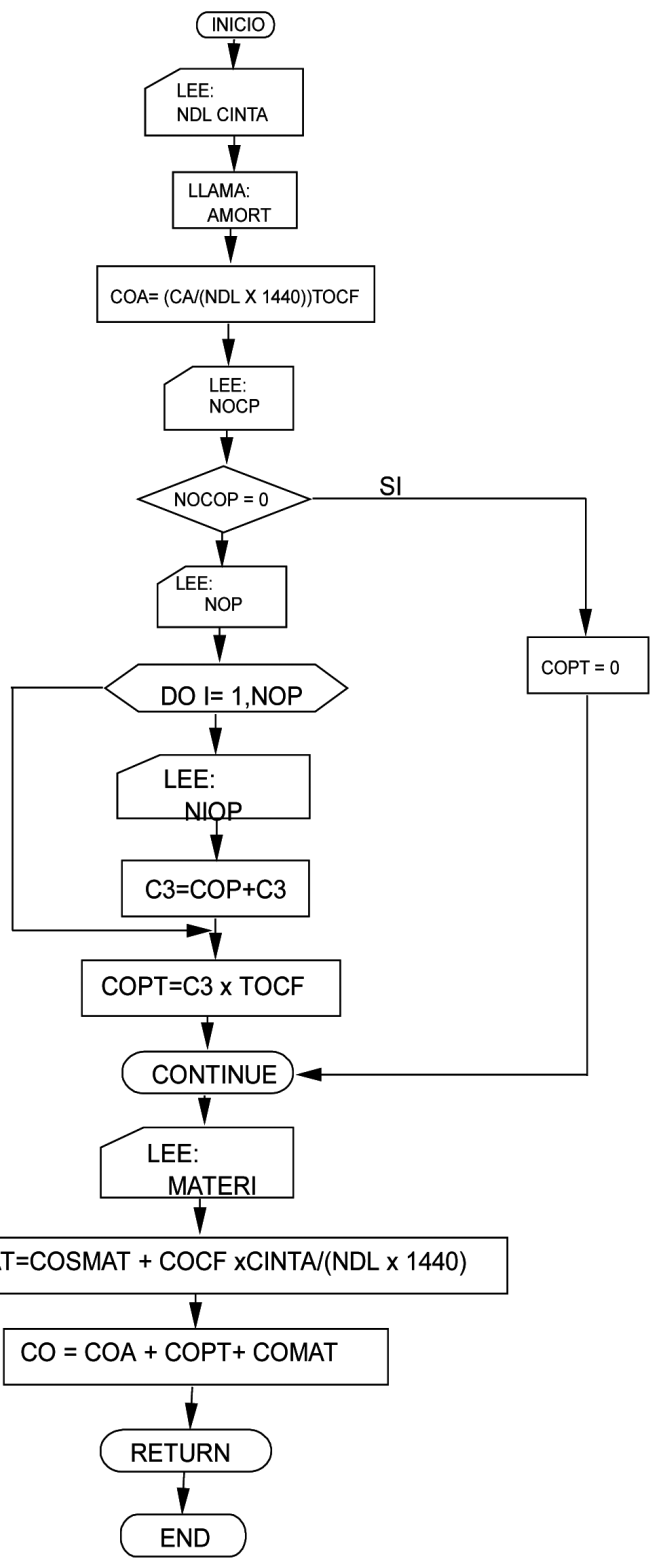

Fig. 4: Diagrama de flujo de la subrutina que estima los costes de oportunidad

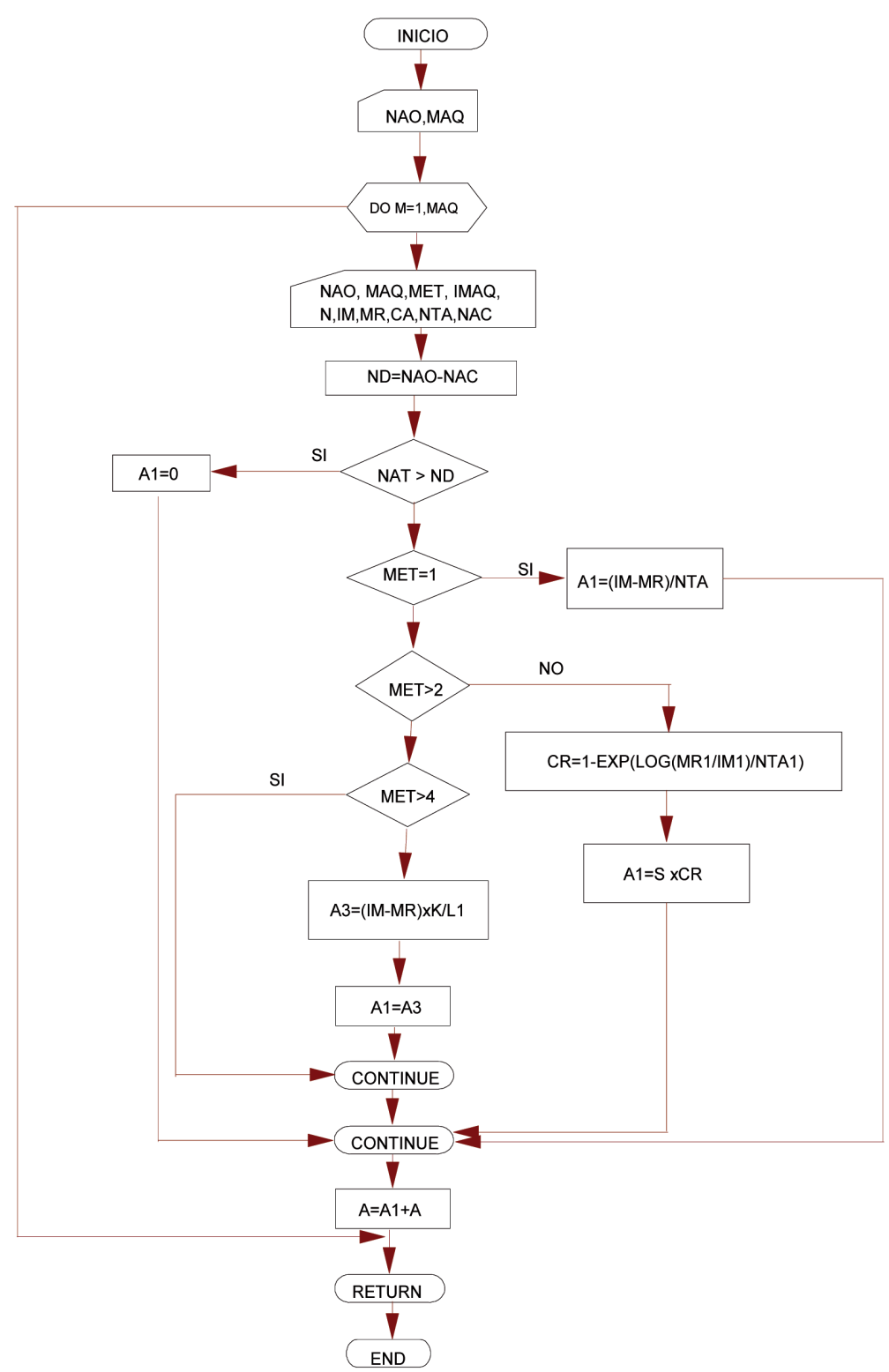

Fig. 5: Diagrama de flujo de la subrutina que estima los costes de amortización 
[2] Cárcel Carrasco F J. "Planteamiento de un modelo de mantenimiento industrial basado en técnicas de gestión del conocimiento". OmniaScience, 2014.DOI: http:// dx.doi.org/10.3926/oms.198.

[3] Shingo S. (1993). "Una revolución en la producción: El sistema SMED". Editorial TGP. 1993. Madrid.

[4] HOYLE, D., THOMPSON, J. Del aseguramiento a la gestión de la calidad: el enfoque basado en procesos. AENOR, 2002, ISBN: 848143-240-7.

[5] Cárcel Carrasco J. (2002). Tesis Doctoral. "Sistema de Transmisión de la experiencia y gestión del conocimiento en la ingeniería del mantenimiento industrial", UNED (2002).

[6] Rodríguez Méndez M. "La seguridad en el proceso de cambio de útiles". Prevención. Julio-Septiembre. 2005. Vol. 173. p 66-77.

[7] Detti P, Meloni C, Pranzo M. "Minimizing and balancing setups in a serial production system". International Journal of Production Research. 2007. Vol 45-24. p 5769-5788. DOI: 10.1080/00207540701636306

[8] Krasik V, Leung J, Pinedo M, Zhang

J. "Scheduling multiple products on parallel machines with setup costs." Naval Research Logistics. October 2008. Vol. 55-7.p 654-669. DOI: 10.1002/nav.20309

[9] Cattrysse D, Salomon M, Kuik R, Luk N., Van Wasenhove "A dual ascent and column generation heuristic for the discrete lot sizing and scheduling problem with setup times". Management Science. April 1993. Vol. 39-4. P. 477-483. DOI: 10.1287/mnsc.39.4.477

[10] Dilts D M, Ramsing K D. "Joint lot sizing and scheduling of multiple items with sequence-dependent setup costs." Decision sciences. March 1989. Vol. 20-1.p 120-133. DOI: 10.1111/j.1540-5915.1989. tb01400.x

[11] Dessouky M. "Using queueing network models to set lot-sizing policies for printed circuit board assembly operations". Production and Inventory Management Journal. 1998.p. 38-43

[12] Khouja M. "The use of minor setups within production cycles to improve product quality and yield." International Transactions in Operational Research. 2005, Vol. 1-42. p. 403-416. DOI: 10.1111/ j.1475-3995.2005.

[13] Mileham A R, Culley S J, Owen G W, McIntosh R I. "Rapid changeover a pre-requisite for responsive manufacture". International Journal of Operations \&t Production Management. 1999. Vol. 19-8. P. 785-796. DOI: 10.1108/01443579910274383

[14] Wang X, Cheng T C E. "Two-machine flowshop scheduling with job class setups to minimize total flowtime." Computers \& Operations Research. November 2005, vol. 32-11. P. 2751 2770. DOI: 10.1016/j.cor.2004.04.002

[15] Darwish M A. "EPQ Models with varying setup cost". International Journal of Production Economics. May 2008.Vol. 113-1. p 297-306. DOI: 10.1016/j.ijpe.2007.07.010

[16] Haase K, Kimms A. "Lot sizing and scheduling with sequencedependent setup costs and times and efficient rescheduling opportunities." International Journal of Production Economics. June 2000. Vol 66-2. P. 159-169.DOI: 10.1016/ S0925-5273(99)00119-X

[17] Rodríguez Méndez, M. "El proceso de cambio de útiles. La flexibilidad de una fábrica". FC Editorial. (2003) Madrid.

[18] Companys-Pascual R, Mateo-Doll $M$, Robas-Vila I. "Programación bicriterio para maquinas en paralelo. Consideración de tiempos de preparación dependientes en la secuencia". DYNA Ingeniería e Industria. Junio 2009. Vol. 84-5 p. $429-440$

[19] Martinelli F, Valigi P. "Dynamic scheduling for a single machine system under different setup and buffer capacity scenarios" Asian Journal of Control. June 2004. Vol. 6-2. p. 229-241. DOI: 10.1111/ j.1934-6093.2004.

[20] Duenyas I, Van Oyen M P. "Stochastic scheduling of parallel queues with set-up costs". Queueing Systems. 1995. Vol. 19. P. 421-444. DOI: 10.1007/BF01151932

[21] Hwang J, Singh M R. "Optimal production policies for multi-stage systems with setup costs and uncertain capacities". Management
Science. September 1998. Vol. 44-9. P. 1279-1294.DOI: 10.1287/ mnsc.44.9.1279

[22] Katok E, Lewis H S, Harrison T P. "Lot sizing in general assembly systems with setup costs, setup times, and multiple constrained resources". Management Science. June 1998. Vol. 44-6. p. 859-877. DOI: $10.1287 \mid$ mnsc.44.6.859.

[23] Gallego G, Moon I. "The effect of externalizing setups in the economic lot scheduling problem". Operations Research. May/June 1992. Vol. 40-3. p. 614-619. DOI: 10.1287/ opre.40.3.614 